\title{
Expressions of Neuregulin $1 \beta$ and ErbB4 in Prefrontal Cortex and Hippocampus of a Rat Schizophrenia Model Induced by Chronic MK-801 Administration
}

\author{
Yu Feng, ${ }^{1}$ Xiao-Dong Wang, ${ }^{1,2}$ Chun-Mei Guo, ${ }^{1}$ Yang Yang, ${ }^{1}$ Ji-Tao Li, ${ }^{1}$ \\ Yun-Ai Su, ${ }^{1}$ and Tian-Mei Si ${ }^{1}$ \\ ${ }^{1}$ Key Laboratory of Mental Health of National Health Ministry, Institute of Mental Health, Peking University, \\ No. 51 Hua Yuan Bei Road, Haidian District, Beijing 100191, China \\ ${ }^{2}$ Max Planck Institute of Psychiatry, Kraepelinstr. 2-10, 80804 Munich, Germany
}

Correspondence should be addressed to Tian-Mei Si, si.tian-mei@163.com

Received 16 January 2010; Accepted 22 February 2010

Academic Editor: Xudong Huang

Copyright (C) 2010 Yu Feng et al. This is an open access article distributed under the Creative Commons Attribution License, which permits unrestricted use, distribution, and reproduction in any medium, provided the original work is properly cited.

\begin{abstract}
Recent human genetic studies and postmortem brain examinations of schizophrenia patients strongly indicate that dysregulation of NRG1 and ErbB4 may be important pathogenic factors of schizophrenia. However, this hypothesis has not been validated and fully investigated in animal models of schizophrenia. In this study we quantitatively examined NRG1 and ErbB4 protein expressions by immunohistochemistry and Western blot in the brain of a rat schizophrenia model induced by chronic administration of MK-801 (a noncompetitive NMDA receptor antagonist). Our data showed that NRG1 $\beta$ and ErbB4 expressions were significantly increased in the rat prefrontal cortex and hippocampus but in different subregions. These findings suggest that altered expressions of NRG1 and ErbB4 might be attributed to the schizophrenia. Further study in the role and mechanism of NRG1 and ErbB4 may lead to better understanding of the pathophysiology for this disorder.
\end{abstract}

\section{Introduction}

Schizophrenia is a complex and severe brain disorder with poorly defined etiology and pathophysiology. Emerging clinical investigations have suggested that there are certain susceptible genes that may be responsible of the pathophysiology of schizophrenia, such as COMT (Catechol-O-methyl transferase), DTNBP1 (Dysbindin), NRG1 (Neuregulin 1), ErbB4, RGS4 (Regulator of G-protein signaling 4), DISC1 (Disrupted-in-schizophrenia 1), and G72 [1-5]. NRG1 and ErbB4 transgenic mice have shown "schizophrenia-like" behavioral deficits, indicating that NRG1 and its receptor ErbB4 might be two of crucial pathogenic factors for the schizophrenia [6-8].

Accumulating reports have demonstrated the significance of NRG1 and ErbB4 function in the brain. For example, NRG1 comprises a family of transmembrane or secreted proteins, sharing an epidermal growth factor(EGF- ) like domain that specifically activates ErbB receptor tyrosine kinases $[9,10]$. Among the ErbB receptors, ErbB4 is the major mediator of NRG1 function in the brain [11, 12]. In the central nervous system, NRG1-ErbB4 signaling regulates neural and glial development [13], axon myelination and ensheathment [14-17], synaptic plasticity [11, 18], expression and activation of neurotransmitter receptors [2, 18], neurotrophic coupling, and neuroprotection following neurological disorders [11].

Importantly, dysregulation and dysfunction of NRG1 and ErbB4 may be associated with some types of neurological disorders [19]. Clinical evidence has revealed altered NRG1 and its receptor ErB4 expressions in postmortem brains of schizophrenia patients $[5,20,21]$. Since the above tested brain samples of all patients received antipsychotic treatment before death, the association of the altered NRG1 and ErbB4 expressions with schizophrenia pathology or antipsychotic drug effects remains unknown. Therefore, it is fundamentally and clinically significant to investigate the role and mechanism of NRG1 and ErB4 in animal models 
of schizophrenia. By doing so, for the first step, in this study we aimed to determine whether there is altered expression of NRG1 and ErbB4 in an animal model of schizophrenia.

Among a number of schizophrenia animal models, a very commonly used rat model is induced by chronic dizocilpine maleate (MK-801, a noncompetitive NMDA receptor antagonist) administration, which was selected in this study $[22,23]$. This model was based on the key pathological mechanism of NMDA glutamatergic hypofunction in schizophrenia [24]. It has been well documented that repeated administration of NMDA receptor antagonists could induce schizophrenia-like behavioral deficits in normal rats $[25,26]$. In this animal model, we examined expressions of NRG1 $\beta$ and ErbB4 proteins in the prefrontal cortex and hippocampus, because the two brain regions are closely related to the pathophysiology of schizophrenia [2729].

\section{Materials and Methods}

2.1. Animals. Adult male Wistar rats (Vital River, Beijing, China) weighing $185-225 \mathrm{~g}$ were group-housed under a 12hour light/dark cycle with ad libitum access to both food and water. All procedures were performed in accordance with the National Institute of Health's Guide for the Use and Care of Laboratory Animals and were approved by the Peking University Committee on Animal Care and Use.

2.2. Drug Treatment. MK-801 (St. Louis, MO, USA) was dissolved with normal saline. After a 1-week acclimatization, the rats were randomly divided into two groups, and each rat received intraperitoneal injection of either MK-801 $(0.5 \mathrm{mg} / \mathrm{kg}, n=10)$ or normal saline (vehicle, $n=10)$ for 4 weeks. The dosage of the MK- 801 used in this study was based on other previous studies [30]. All rats received single, daily injection between 09:00 and 11:00 am.

2.3. Immunohistochemistry. The rats ( $n=5$ per group) were deeply anaesthetized with chloral hydrate $(300 \mathrm{mg} / \mathrm{kg})$ and transcardially perfused with $250 \mathrm{~mL}$ cool saline solution followed by $300 \mathrm{~mL}$ of $4 \%$ paraformaldehyde in $0.1 \mathrm{M}$ phosphate buffered saline (PBS, pH 7.4). Brains were immediately removed, postfixed in the same fixative overnight, and cryoprotected in $30 \%$ sucrose at $4{ }^{\circ} \mathrm{C}$ for $5 \mathrm{~d}$. After quenching by immersing them in cold $\mathrm{N}$-hexane on dry ice, the brains were stored at $-80^{\circ} \mathrm{C}$ until analyzed.

Serial coronal sections of $20 \mu \mathrm{m}$ thickness at various levels $(100 \mu \mathrm{m}$ interval) through the prefrontal cortex (bregma $3.72-2.52$ ) and hippocampus (bregma -2.76 to -3.96 ) [31] were cut on a cryostat (Leica, Wetzlar, Germany) and slide-mounted. After blocking in $10 \%$ normal goat serum in $0.1 \mathrm{M}$ PBS containing $0.3 \%$ Triton X-100 for 1 hour at room temperature (RT), sections were incubated with NRG1 $\beta$ - (sc-347) or ErbB4- (sc-283, all from Santa Cruz Biotechnology Inc., Santa Cruz, CA, USA) specific antibodies diluted $1: 100$ in $0.1 \mathrm{M}$ PBS overnight at $4^{\circ} \mathrm{C}$. The next morning, sections were incubated with primary antibodies for an additional 1 hour at RT, followed by incubation with biotinylated secondary antibodies (Santa Cruz Biotechnology Inc.) for 3 hours at RT, and then incubated with avidin-biotin-horseradish peroxidase (Santa Cruz Biotechnology Inc.) for 3 hours at RT. Finally, the sections were developed with a solution of $50 \% 3,3^{\prime}$ diaminobenzidine (DAB) and 3\% hydrogen peroxide in $0.1 \mathrm{M}$ PBS, dehydrated, and coverslipped.

Four sections ( $20 \mu \mathrm{m}$ thickness, $100 \mu \mathrm{m}$ interval) from the prefrontal cortex and hippocampus were obtained and immunoreactivity was determined and quantitated as previously described [32]. Briefly, immunoreactive staining was observed and photographed at $\times 40$ magnification using an Olympus BX51 microscope fitted with a CoolSNAP MP5 CCD camera (Roper Scientific, Tucson, AZ, USA). Images were converted to grey scale and analyzed with Image Pro-Plus software (Media Cybernetics, Silver Spring, MD, USA). For calculating immunoreactivity of prefrontal cortex, a $1 \times 1 \mathrm{~mm}^{2}$ cursor was placed on the prelimbic cortex (PrL) or the cingulate cortex area $1(\mathrm{Cg} 1)$ plus secondary motor cortex (M2) [31]. Constant background intensity across different sections from each animal was set so that positivelabeled cells were selected only if they reached a defined threshold above background, then the integrated optical density (IOD $=$ immunoreactive area $\times$ average optical density) values of $\operatorname{PrL}$ and $\mathrm{Cg} 1$ plus M2 were measured and designated as optical density (OD). Optical density value of hippocampal subregions and background were measured by placing several adjacent circular cursors with a diameter of $100 \mu \mathrm{m}$ along the pyramidal cell layer of CA1 ( 8 cursors) and CA3 (12 cursors), the granule cell layer of DG ( 8 cursors), and corpus callosum (CC, 8 cursors). Optical density values of corpus callosum were designated as background. The differences of value between the target and background were calculated and designated as OD. All results were normalized by taking the value of the vehicle group as $100 \%$.

2.4. Western Blot. After sacrificing animals, the brains were rapidly removed and dissected to obtain the prefrontal cortex and hippocampus [33]. Tissue from individual rats was immediately homogenized on ice in ice-cold lysis buffer (137 mM NaCl, $20 \mathrm{mM}$ Tris-HCl (pH 8.0), 1\% NP-40, $10 \%$ glycerol, $1 \mathrm{mM}$ PMSF, $10 \mathrm{mg} / \mathrm{mL}$ aprotinin, $1 \mathrm{mg} / \mathrm{mL}$ leupeptin, and $0.5 \mathrm{mM}$ sodium vanadate), sonicated, and centrifuged. The supernatants were stored at $-80^{\circ} \mathrm{C}$ until assayed.

Samples containing $20 \mu \mathrm{g}$ of protein were resolved by 7.5\% (for detection of ErbB4 receptor) or 10\% (for NRG1 $\beta$ isoforms) SDS-PAGE gels. Immunoblotting was conducted with NRG1 $\beta$ - (sc-347, $1: 1000)$ or ErbB4- (sc-283, $1: 1000)$ specific antibodies (all from Santa Cruz Biotechnology Inc.). We stripped and reprobed bolts with rabbit antiactin polyclonal antibody (Santa Cruz Biotechnology Inc.). The immunoreactive signals of NRG1 $\beta$ and ErbB4 were quantified by densitometry and the values were corrected based on their corresponding actin levels. All results were normalized by taking the value of the vehicle group as $100 \%$. 
TABLE 1: Immunoreactivity of NRG1 $\beta$ and ErbB4 in the rat prefrontal cortex and hippocampal subregions.

\begin{tabular}{ccccc}
\hline & & & Vehicle & MK-801 \\
\hline PFC & NRG1 $\beta$ & Cg1 and M2 & $100 \pm 6.12$ & $101.84 \pm 6.69$ \\
& & PrL & $100 \pm 4.79$ & $108.75 \pm 5.09$ \\
& \multirow{2}{*}{ ErbB4 } & Cg1 and M2 & $100 \pm 7.96$ & $125.33 \pm 7.45$ \\
& & PrL & $100 \pm 6.08$ & $135.09 \pm 8.03^{*}$ \\
\hline HC & NRG1 $\beta$ & CA1 & $100 \pm 10.79$ & $204.08 \pm 10.87^{*}$ \\
& & CA3 & $100 \pm 7.69$ & $142.92 \pm 6.39^{*}$ \\
& & DG & $100 \pm 10.29$ & $167.18 \pm 11.02^{*}$ \\
& \multirow{2}{*}{ ErbB4 } & CA1 & $100.00 \pm 6.28$ & $140.23 \pm 8.66^{*}$ \\
& & CA3 & $100.00 \pm 4.87$ & $106.27 \pm 5.94$ \\
& & DG & $100.00 \pm 7.48$ & $106.32 \pm 7.04$ \\
\hline
\end{tabular}

PFC: prefrontal cortex; HC: hippocampus; Cg1: cingulate cortex area 1; M2: secondary motor cortex; PrL: prelimbic cortex; DG: dentate gyrus.

${ }^{*} P<.05$ compared to vehicle group.

2.5. Antibody Specificity. The specificity of antibodies was examined by Western blot as previously described [32]. The signal of the $65 \mathrm{kDa}$ NRG1 $\beta$ isoform was reduced and labeling of the other NRG1 $\beta$ isoforms and ErbB4 isoforms was eliminated by preabsorption with corresponding antigen peptides (Santa Cruz Biotechnology Inc.). Antibodies against actin generated one main band.

2.6. Statistical Analysis. All data were expressed as the means \pm S.E.M. Statistical differences between groups were determined by independent samples $t$-test. Differences of $P<.05$ were considered to be significant.

\section{Results}

3.1. Changes of NRG1 $\beta$ and ErbB4 Protein Expressions in the Prefrontal Cortex. Protein samples extracted from rat prefrontal cortex were analyzed by Western blot. Quantitative data showed that expressions of all five NRG1 $\beta$ isoforms were significantly elevated with relative levels (\% of vehicle treated controls) of $220 \pm 5$ for $35 \mathrm{kDa}$ isoform, $245 \pm 22$ for $65 \mathrm{kDa}$ isoform, $255 \pm 23$ for $80 \mathrm{kDa}$ isoform, $266 \pm$ 10 for $85 \mathrm{kDa}$ isoform, and $224 \pm 18$ for $95 \mathrm{kDa}$ isoform, respectively, while all three isoforms of ErbB4 were also significantly upregulated with relative levels ( $\%$ of vehicle treated controls) of $190 \pm 26$ for $60 \mathrm{kDa}$ isoform, $164 \pm$ 5 for $120 \mathrm{kDa}$ isoform, and $199 \pm 12$ for $185 \mathrm{kDa}$ isoform, respectively (Figures $1(\mathrm{a})-1(\mathrm{~d})$ ).

Immunohistochemistry was applied to localize and quantitate NRG1 $\beta$ and ErbB4 immunoreactivity in different subregions of prefrontal cortex (Figures 1(e)-1(h) and Table 1). Quantification of immunoreactive intensity showed slight increases of NRG1 $\beta$ expression in the two cortex subregions of prelimbic cortex (PrL) and the cingulate cortex area 1 plus secondary motor cortex ( $\mathrm{Cg} 1$ and M2), but did not reach statistic significance. However, the immunoreactivity of ErbB4 was significantly increased in the PrL region, while no significant difference was observed in $\mathrm{Cg} 1$ and $\mathrm{M} 2$ region.
From the DAB-stained morphology of immunopositive cells, it is noticed that they are mainly neuron-like cells [32].

\subsection{Changes of NRG1 $\beta$ and ErbB4 Protein Expressions in the} Hippocampus. Western blot analysis revealed that expressions of three NRG1 $\beta$ isoforms were significantly elevated with relative levels (\% of vehicle treated controls) of 169 \pm 7 for $35 \mathrm{kDa}$ isoform, $159 \pm 17$ for $65 \mathrm{kDa}$ isoform, and $134 \pm 12$ for $95 \mathrm{kDa}$ isoform, respectively; while all three isoforms of ErbB4 were also significantly upregulated with relative levels (\% of vehicle treated controls) of $330 \pm 28$ for $60 \mathrm{kDa}$ isoform, $221 \pm 15$ for $120 \mathrm{kDa}$ isoform, and $235 \pm 26$ for $185 \mathrm{kDa}$ isoform, respectively (Figures $2(\mathrm{a})-2(\mathrm{~d})$ ).

Consistent with Western blot results, immunostaining analysis revealed significant increases of NRG1 $\beta$ immunoreactivity in the pyramidal cell layer of CA1 and CA3 and in the granule cell layer of the dentate gyrus (Figures 2(e)2 (j) and Table 1). Similar to the results for NRG1 $\beta$, MK801 increased expression of ErbB4 receptor in the CA1 subregion, while there were no significant differences that can be detected in CA3 and the dentate gyrus. From the DABstained morphology of immunopositive cells, it ia noticed that they are mainly neuron-like cells [32].

\section{Discussion}

Our major findings from this study were that in this schizophrenia animal model induced by chronic MK-801 administration, Western blot results showed significantly increased protein levels of NRG1 in the rat prefrontal cortex, while immunohistochemistry analysis observed the increase of NRG1 immunoreactivity in the PrL and the Cg1 and M2 regions, but it did not reach statistic significance. This discrepancy results between Western blot and immuohistochemistry may be due to the different sensitivity of these two assessments and small sample size. Additionally, in this study, we found that protein level of ErbB4 is upregulated in the rat prefrontal cortex by Western blot, while the immunoreactivity of ErbB4 only increased in the PrL region. Furthermore, protein levels of both NRG1 and ErbB4 were increased in rat hippocampus, but the increased immunoreactivities of the two proteins were located in different subregions. Significantly increased NRG1 immuoreactivity can be seen in the pyramidal cell layer of CA1 and CA3 and in the granule cell layer of the dentate gyrus, while ErbB4 was only significantly increased in CA1 subregion. There might be important role and mechanisms beyond the distinguishable expression patterns of NRG1 and ErbB4 in this schizophrenia model which need further investigations.

Our findings are consistent with the clinical observations of dysregulated expression of NRG1 and ErbB4 in postmortem brain of schizophrenia patients $[20,21]$. Interestingly, our previous study found that changes of NRG1 and ErbB4 protein levels can be induced by chronic administration of antipsychotic drugs in normal rat prefrontal cortex and hippocampus [32], indicating that regulations of NRG1 and ErbB4 proteins may be associated 


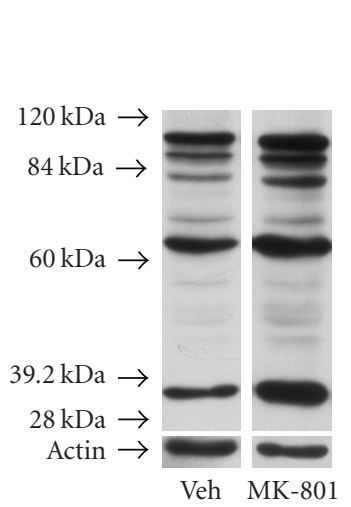

(a)

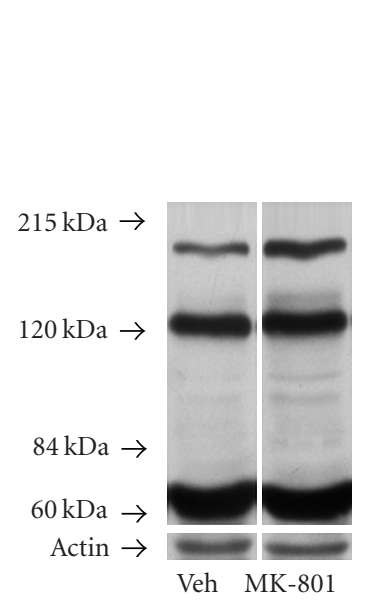

(c)

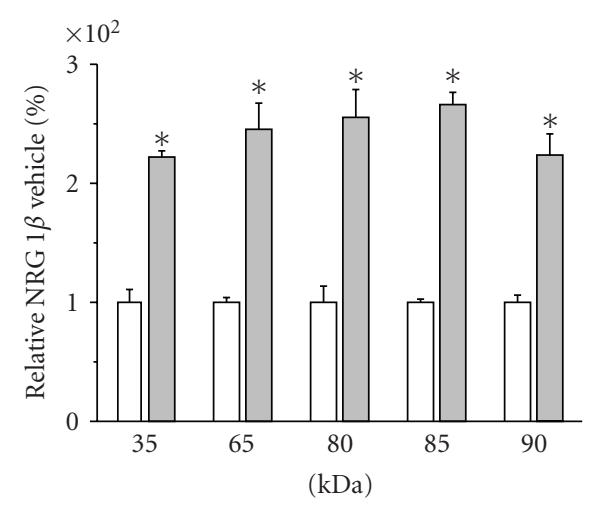

(b)

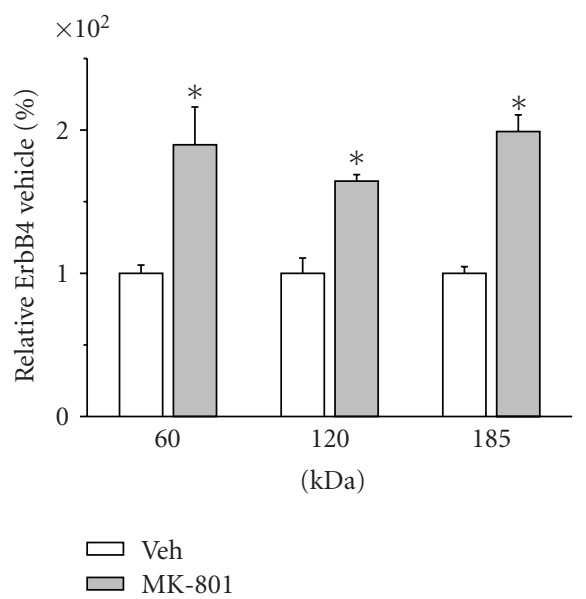

(d)

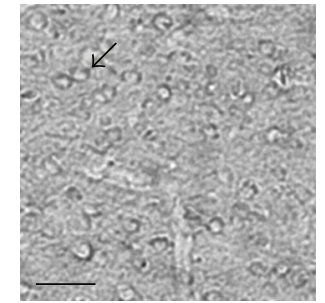

(e)

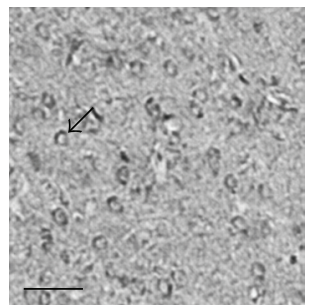

(g)

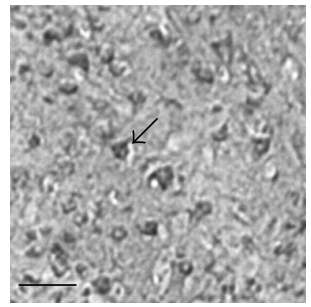

(f)

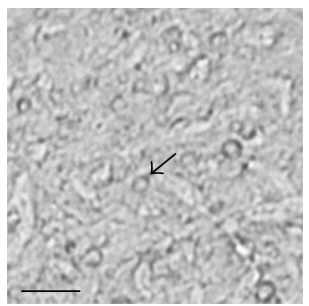

(h)

FIGURE 1: Expression of NRG1 $\beta$ and ErbB4 proteins in the rat prefrontal cortex. (a) Representative Western blots of NRG1 $\beta$ protein isoforms. (b) Quantitative analysis of NRG1 $\beta$ immunoblots. (c) Representative Western blots of ErbB4 proteins isoforms. (d) Quantitative analysis of ErbB4 immunoblots. The data were normalized by taking the value of vehicle group as $100 \%$ and expressed as means \pm S.E.M. $(n=5) .{ }^{*} P<.05$. (e) Representative section of ErbB4 immunohistochemistry in the PrL region of prefrontal cortex from vehicle group. (f) Representative section of ErbB4 immunohistochemistry in the PrL region of prefrontal cortex from MK-801-treated group. (g) Representative section of ErbB4 immunohistochemistry in the Cg1 and M2 region of prefrontal cortex from vehicle group. (h) Representative section of ErbB4 immunohistochemistry in the Cg1 and M2 region of prefrontal cortex from MK-801-treated group. Arrows indicate ErbB4expressing neurons in the prefrontal cortex. Scale bar for (e)-(h): $50 \mu \mathrm{m}$. 


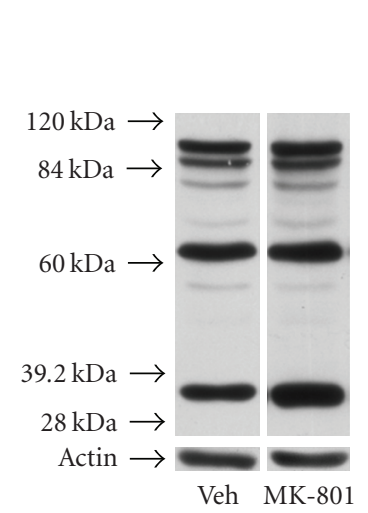

(a)

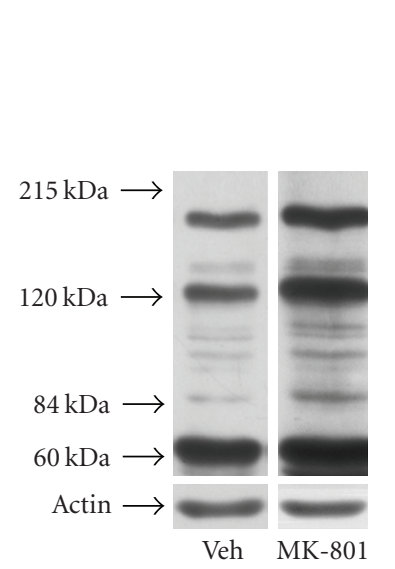

(c)

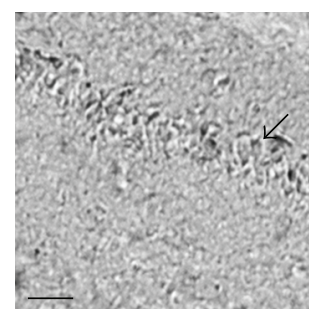

(e)

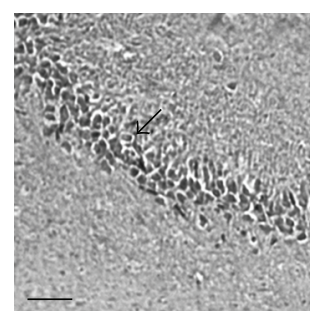

(h)

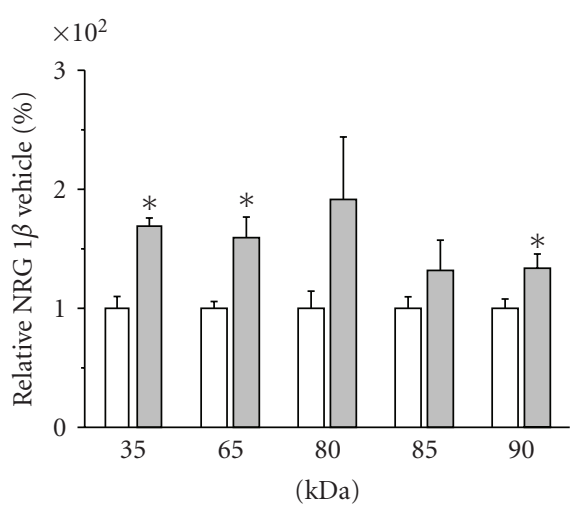

(b)

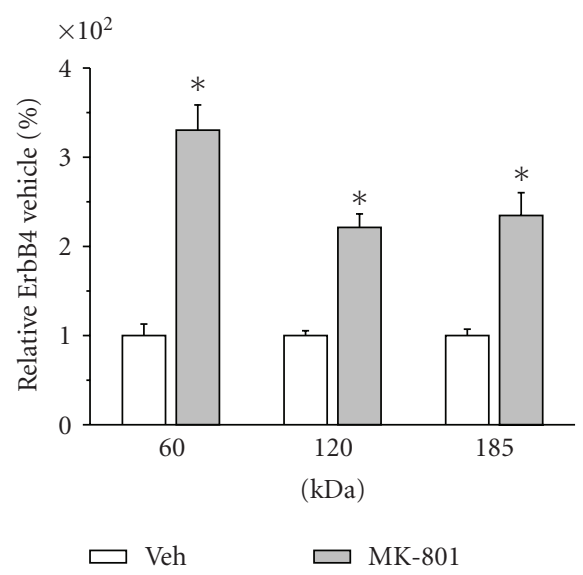

(d)

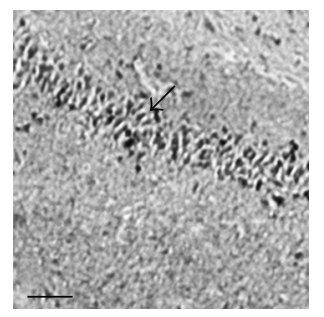

(f)

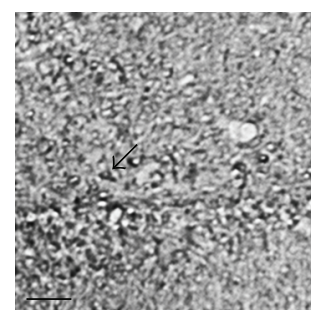

(i)

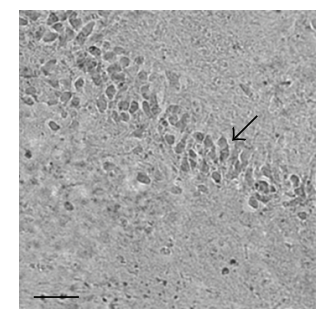

(g)

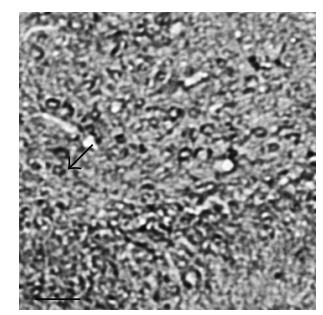

(j)

FIGURE 2: Expression of NRG1 $\beta$ and ErbB4 proteins in the rat hippocampus. (a) Representative Western blots of NRG1 $\beta$ protein isoforms. (b) Quantitative analysis of NRG1 $\beta$ immunoblots. (c) Representative Western blots of ErbB4 protein isoforms. (d) Quantitative analysis of ErbB4 immunoblots. The data were normalized by taking the value of vehicle group as $100 \%$ and expressed as means \pm S.E.M. $(n=5)$. ${ }^{*} P<.05$. (e) Representative section of NRG1 $\beta$ immunohistochemistry in the hippocampal CA1 region from vehicle group. (f) Representative section of NRG1 $\beta$ immunohistochemistry in the hippocampal CA1 region from MK-801-treated group. (g) Representative section of NRG1 $\beta$ immunohistochemistry in the hippocampal CA3 region from vehicle group. (h) Representative section of NRG1 $\beta$ immunohistochemistry in the hippocampal CA3 region from MK-801-treated group. (i) Representative section of NRG1 $\beta$ immunohistochemistry in the hippocampal DG region from vehicle group. (j) Representative section of NRG1 $\beta$ immunohistochemistry in the hippocampal DG region from MK-801treated group. Arrows indicate NRG1-expressing neurons in the hippocampus. Scale bar for (e)-(j): $50 \mu \mathrm{m}$. 
with pathophysiological cascades and therapeutic outcomes of schizophrenia.

Although the roles and mechanisms of the increased NRG1 and ErbB4 in this rat schizophrenia model and correlation to the schizophrenia pathophysiology are incompletely understood from this study, the potential roles and mechanisms might be speculated. Our data showed that repeated blockade of NMDA receptor increased the expression of NRG1 and ErbB4, suggesting that changes of both NRG1 and ErbB4 expressions are associated with NMDA receptor function in this rat model for schizophrenia. Perhaps this is due to the fact that ErbB4 colocalizes with NMDA receptors at postsynaptic sites through interaction with PDZ domain-containing proteins, presuming that there is cross-talk between NMDA receptor and NRG1-ErbB4 signaling $[20,34,35]$.

We are aware that there are several limitations in this short study. We did not study the time course of NRG1 and ErbB4 expression and correlation to the late behavioral deficits, which need to be performed in the future. Since we only tested this rat schizophrenia animal model, we need to know whether increased expression of NRG1 and ErbB4 in other schizophrenia animal models is a common pathological phenomenon of schizophrenia. Except the protein expression changes, we also need to know the function changes of NRG1-ErbB4 signaling, in terms of better understanding their roles and mechanisms. The cellular sources and subcellular distributions of both NRG1 and ErbB4 need to be carefully defined with double staining techniques. Lastly, genetic alteration and pharmacological approaches for manipulating NRG1 and ErbB4 expressions can be applied to further investigate their pathological importance and therapeutic potentials in schizophrenia.

\section{Conclusion}

In summary, our present study indicates that protein levels of NRG1 $\beta$ and ErbB4 in the prefrontal cortex and hippocampus are increased in this rat model of schizophrenia induced by repeated MK-801 administration. These findings suggest that altered expressions of NRG1 and ErbB4 might attribute to the schizophrenia. Further study of the role and mechanism of NRG1 and ErbB4 may lead to better understanding of the pathophysiology of schizophrenia.

\section{Acknowledgment}

This work was supported by National Natural Scientific Foundation of China (Grant no.: 30770775 and 30800361).

\section{References}

[1] P. J. Harrison and M. J. Owen, "Genes for schizophrenia? Recent findings and their pathophysiological implications," The Lancet, vol. 361, no. 9355, pp. 417-419, 2003.

[2] P. J. Harrison and D. R. Weinberger, "Schizophrenia genes, gene expression, and neuropathology: on the matter of their convergence," Molecular Psychiatry, vol. 10, no. 1, pp. 40-68, 2005.
[3] A. J. Law, J. E. Kleinman, D. R. Weinberger, and C. S. Weickert, "Disease-associated intronic variants in the ErbB4 gene are related to altered ErbB4 splice-variant expression in the brain in schizophrenia," Human Molecular Genetics, vol. 16, no. 2, pp. 129-141, 2007.

[4] N. Norton, V. Moskvina, D. W. Morris, et al., "Evidence that interaction between neuregulin 1 and its receptor erbB4 increases susceptibility to schizophrenia," American Journal of Medical Genetics Part B, vol. 141B, no. 1, pp. 96-101, 2006.

[5] G. Silberberg, A. Darvasi, R. Pinkas-Kramarski, and R. Navon, "The involvement of ErbB4 with schizophrenia: association and expression studies," American Journal of Medical Genetics Part B, vol. 141B, no. 2, pp. 142-148, 2006.

[6] R. Gerlai, P. Pisacane, and S. Erickson, "Heregulin, but not ErbB2 or ErbB3, heterozygous mutant mice exhibit hyperactivity in multiple behavioral tasks," Behavioural Brain Research, vol. 109, no. 2, pp. 219-227, 2000.

[7] M. S. Golub, S. L. Germann, and K. C. K. Lloyd, "Behavioral characteristics of a nervous system-specific erbB4 knock-out mouse," Behavioural Brain Research, vol. 153, no. 1, pp. 159170, 2004.

[8] H. Stefansson, E. Sigurdsson, V. Steinthorsdottir, et al., "Neuregulin 1 and susceptibility to schizophrenia," American Journal of Human Genetics, vol. 71, no. 4, pp. 877-892, 2002.

[9] G. D. Fischbach and K. M. Rosen, "ARIA: a neuromuscular junction neuregulin," Annual Review of Neuroscience, vol. 20, pp. 429-458, 1997.

[10] A. Buonanno and G. D. Fischbach, "Neuregulin and ErbB receptor signaling pathways in the nervous system," Current Opinion in Neurobiology, vol. 11, no. 3, pp. 287-296, 2001.

[11] L. Mei and W.-C. Xiong, "Neuregulin 1 in neural development, synaptic plasticity and schizophrenia," Nature Reviews Neuroscience, vol. 9, no. 6, pp. 437-452, 2008.

[12] G. Carpenter, "ErbB-4: mechanism of action and biology," Experimental Cell Research, vol. 284, no. 1, pp. 66-77, 2003.

[13] G. Lemke, "Glial control of neuronal development," Annual Review of Neuroscience, vol. 24, pp. 87-105, 2001.

[14] A. N. Garratt, S. Britsch, and C. Birchmeier, "Neuregulin, a factor with many functions in the life of a Schwann cell," BioEssays, vol. 22, no. 11, pp. 987-996, 2000.

[15] G. V. Michailov, M. W. Sereda, B. G. Brinkmann, et al., "Axonal neuregulin-1 regulates myelin sheath thickness," Science, vol. 304, no. 5671, pp. 700-703, 2004.

[16] K.-A. Nave and J. L. Salzer, "Axonal regulation of myelination by neuregulin 1," Current Opinion in Neurobiology, vol. 16, no. 5, pp. 492-500, 2006.

[17] C. Taveggia, G. Zanazzi, A. Petrylak, et al., "Neuregulin-1 type III determines the ensheathment fate of axons," Neuron, vol. 47, no. 5, pp. 681-694, 2005.

[18] S. E. Arnold, K. Talbot, and C.-G. Hahn, "Neurodevelopment, neuroplasticity, and new genes for schizophrenia," Progress in Brain Research, vol. 147, pp. 319-345, 2004.

[19] R. M. Esper, M. S. Pankonin, and J. A. Loeb, "Neuregulins: versatile growth and differentiation factors in nervous system development and human disease," Brain Research Reviews, vol. 51, no. 2, pp. 161-175, 2006.

[20] C.-G. Hahn, H.-Y. Wang, D.-S. Cho, et al., "Altered neuregulin 1-erbB4 signaling contributes to NMDA receptor hypofunction in schizophrenia," Nature Medicine, vol. 12, no. 7, pp. 824-828, 2006.

[21] V. Z. Chong, M. Thompson, S. Beltaifa, M. J. Webster, A. J. Law, and C. S. Weickert, "Elevated neuregulin-1 and ErbB4 protein in the prefrontal cortex of schizophrenic patients," Schizophrenia Research, vol. 100, no. 1-3, pp. 270-280, 2008. 
[22] D. Rujescu, A. Bender, M. Keck, et al., "A pharmacological model for psychosis based on N-methyl-D-aspartate receptor hypofunction: molecular, cellular, functional and behavioral abnormalities," Biological Psychiatry, vol. 59, no. 8, pp. 721729, 2006.

[23] D. Kondziella, E. Brenner, E. M. Eyjolfsson, K. R. Markinhuhta, M. L. Carlsson, and U. Sonnewald, "Glial-neuronal interactions are impaired in the schizophrenia model of repeated MK801 exposure," Neuropsychopharmacology, vol. 31, no. 9, pp. 1880-1887, 2006.

[24] J. W. Olney and N. B. Farber, "Glutamate receptor dysfunction and schizophrenia," Archives of General Psychiatry, vol. 52, no. 12, pp. 998-1007, 1995.

[25] J. D. Jentsch and R. H. Roth, "The neuropsychopharmacology of phencyclidine: from NMDA receptor hypofunction to the dopamine hypothesis of schizophrenia," Neuropsychopharmacology, vol. 20, no. 3, pp. 201-225, 1999.

[26] G. E. Duncan, S. Miyamoto, J. N. Leipzig, and J. A. Lieberman, "Comparison of brain metabolic activity patterns induced by ketamine, MK- 801 and amphetamine in rats: support for NMDA receptor involvement in responses to subanesthetic dose of ketamine," Brain Research, vol. 843, no. 1-2, pp. 171183, 1999.

[27] D. R. Weinberger, M. S. Aloia, T. E. Goldberg, and K. F. Berman, "The frontal lobes and schizophrenia," Journal of Neuropsychiatry and Clinical Neurosciences, vol. 6, no. 4, pp. 419-427, 1994.

[28] B. Elvevag and T. E. Goldberg, "Cognitive impairment in schizophrenia is the core of the disorder," Critical Reviews in Neurobiology, vol. 14, no. 1, pp. 1-21, 2000.

[29] D. P. Eisenberg and K. F. Berman, "Executive function, neural circuitry, and genetic mechanisms in schizophrenia," Neuropsychopharmacology, vol. 35, no. 1, pp. 258-277, 2010.

[30] M. S. Seo, S. H. Kim, Y. M. Ahn, et al., "The effects of repeated administrations of MK-801 on ERK and GSK-3 $\beta$ signalling pathways in the rat frontal cortex," International Journal of Neuropsychopharmacology, vol. 10, no. 3, pp. 359-368, 2007.

[31] G. Paxinos and C. Watson, The Rat Brain in Stereotaxic Coordinate, Academic Press, San Diego, Calif, USA, 2005.

[32] X.-D. Wang, Y.-A. Su, C.-M. Guo, Y. Yang, and T.-M. $\mathrm{Si}$, "Chronic antipsychotic drug administration alters the expression of neuregulin 1 $\beta$, ErbB2, ErbB3, and ErbB4 in the rat prefrontal cortex and hippocampus," International Journal of Neuropsychopharmacology, vol. 11, no. 4, pp. 553-561, 2008.

[33] D. A. Gearhart, M.-L. Middlemore, and A. V. Terry, "ELISA methods to measure cholinergic markers and nerve growth factor receptors in cortex, hippocampus, prefrontal cortex, and basal forebrain from rat brain," Journal of Neuroscience Methods, vol. 150, no. 2, pp. 159-173, 2006.

[34] R. A. G. Garcia, K. Vasudevan, and A. Buonanno, "The neuregulin receptor ErbB-4 interacts with PDZ-containing proteins at neuronal synapses," Proceedings of the National Academy of Sciences of the United States of America, vol. 97, no. 7, pp. 3596-3601, 2000.

[35] Y. Z. Huang, S. Won, D. W. Ali, et al., "Regulation of neuregulin signaling by PSD-95 interacting with ErbB4 at CNS synapses," Neuron, vol. 26, no. 2, pp. 443-455, 2000. 

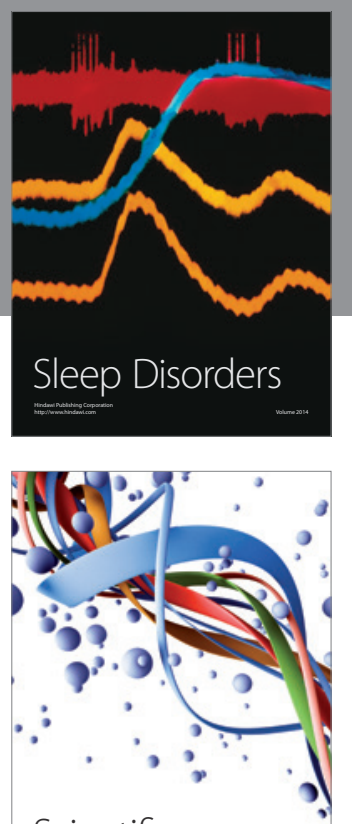

Scientifica
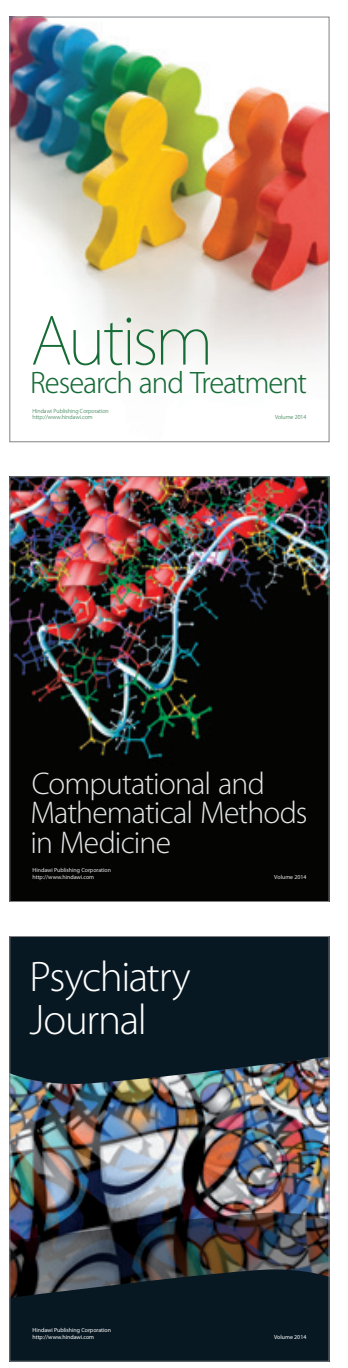
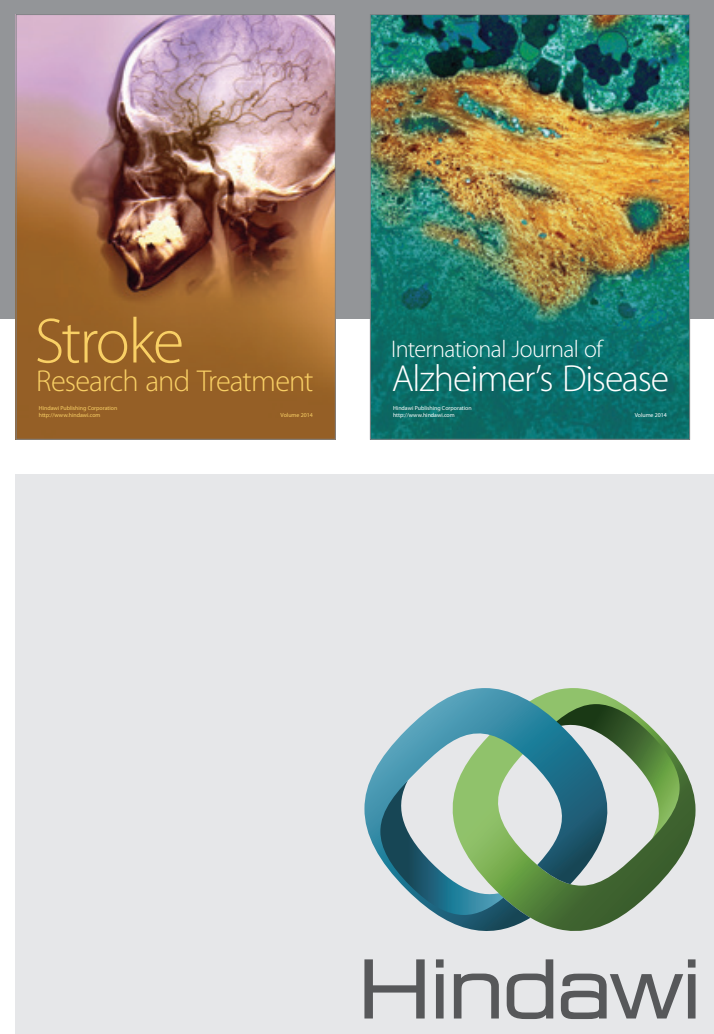

Submit your manuscripts at

http://www.hindawi.com
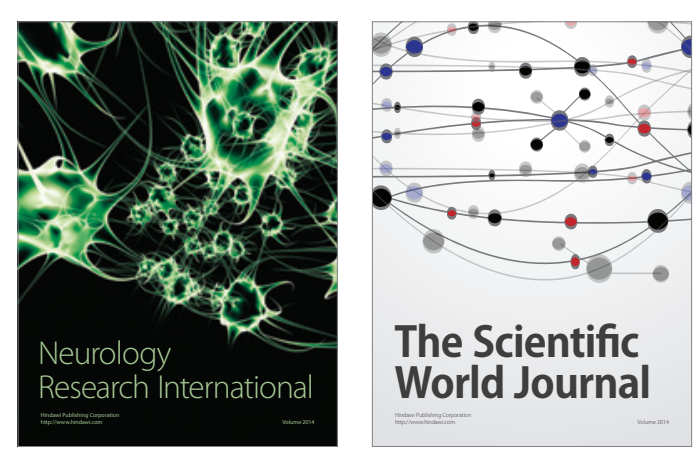

The Scientific World Journal

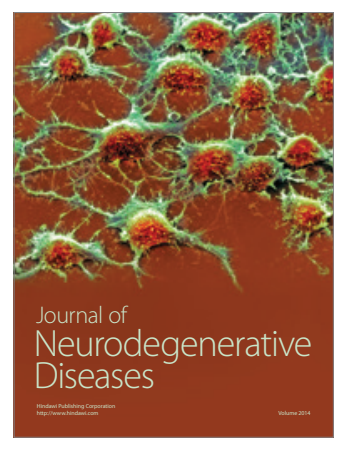

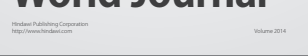

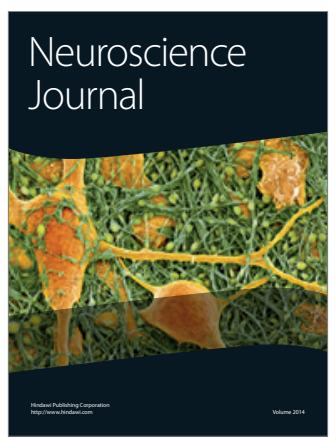

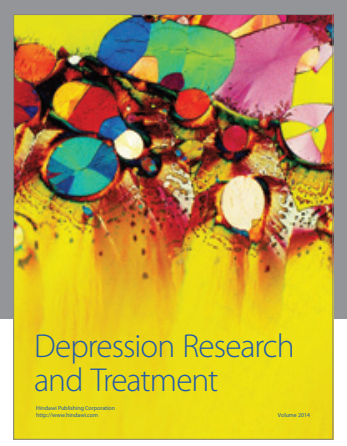
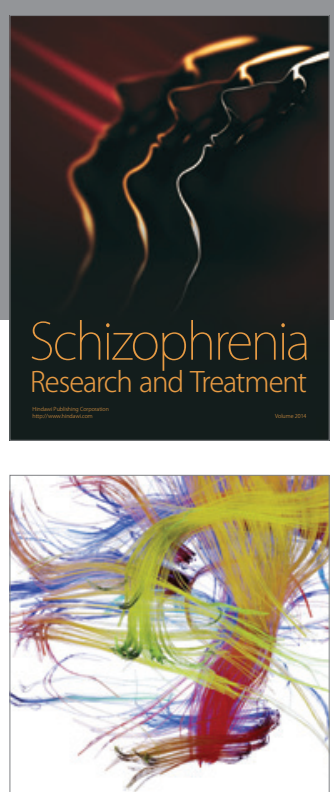

Brain Science

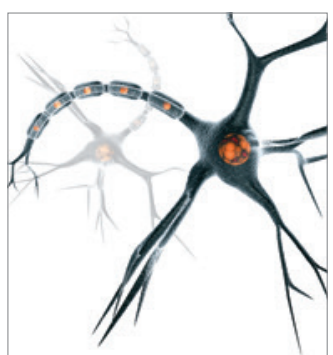

Neural Plasticity
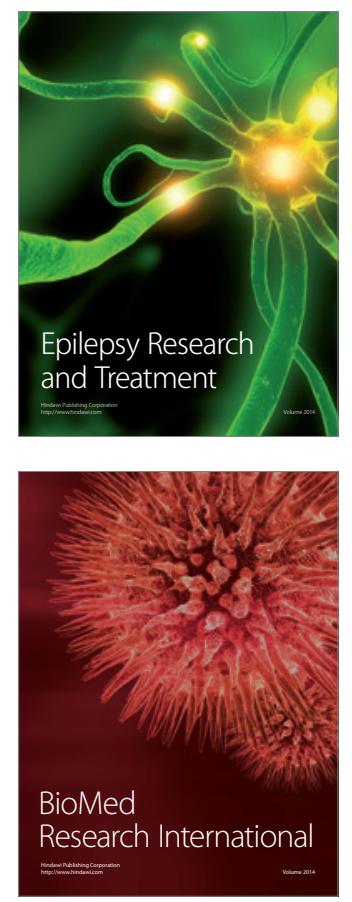

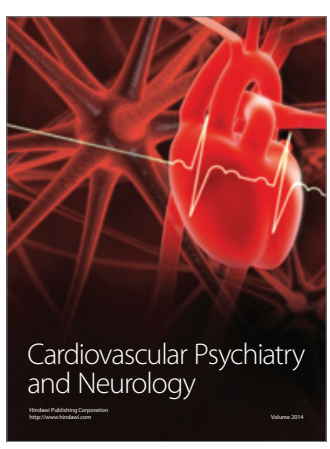

Parkinson's

Disease
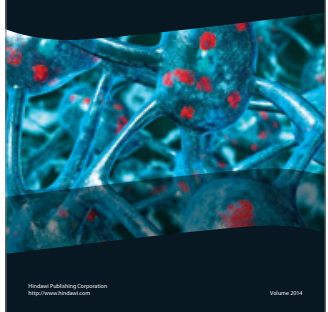